\title{
Hereditary haemorrhagic telangiectasia in a patient taking anticoagulant drugs who has sustained facial trauma
}

\author{
Bartłomiej Kamiński ${ }^{1}$, Katarzyna Błochowiak², Karolina Olek-Hrab ${ }^{3}$, Jerzy Sokalski ${ }^{4}$, Henryk Witmanowski ${ }^{4,5}$
}

1Department of Otolaryngology, District Hospital, Skarzysko-Kamienna, Poland

Head: Bartłomiej Kamiński MD

2Department of Oral Surgery, Poznan University of Medical Sciences, Poland

Head: Prof. Jerzy Sokalski DDS, PhD

${ }^{3}$ Department of Dermatology, Poznan University of Medical Sciences, Poland

Head: Prof. Zygmunt Adamski MD, PhD

4Department of Physiology, Poznan University of Medical Sciences, Poland

Head: Prof. Hanna Krauss MD, PhD

5Department of Plastic, Reconstructive and Aesthetic Surgery, Nicolaus Copernicus University, Collegium Medicum, Bydgoszcz, Poland Head: Prof. Henryk Witmanowski MD, PhD

Postep Derm Alergol 2013; XXX, 3: 199-202 DOI: 10.5114/pdia.2013.35625

\begin{abstract}
The authors present the case of a 41-year-old patient with hereditary hemorrhagic telangiectasia (HHT), who in the past had an aortic valve replacement surgery, currently takes anticoagulant drugs and has sustained an extensive trauma to the nose as a result of a dog bite. The HHT is diagnosed basing on the presence of at least three out of four symptoms or signs: spontaneous epistaxis, vascular lesions in the internal organs, skin telangiectasias and a family history of the disease. The presented patient showed hepatic angioma, history of recurrent bleeding from the tongue and spontaneous epistaxis as well as numerous skin telangiectasias. In his case, HHT coincided with chronic treatment with coagulants implemented after an implantation of the artificial aortic replacement valve, what substantially modified the clinical picture and course of treatment.
\end{abstract}

Key words: hereditary hemorrhagic telangiectasia, anticoagulants, Rendu-Osler-Weber syndrome.

\section{Case report}

A 41-year-old patient with hereditary familial telangiectasia and post-haemorrhagic anaemia was admitted as an emergency case to the Department of Otolaryngology of the District Hospital in Skarzysko-Kamienna. The reason for admission was trauma to the nose resulting from a dog bite. Clinical examination showed a massive haemorrhage from numerous wounds of the face and the nasal cavity. Haemorrhage from the nasal cavity was caused by an extensive injury involving tearing off the left nasal ala and an incomplete tear of the inferior left nasal concha. After bleeding was arrested with electro coagulation and pressure, plastic surgery of the nose was performed. Anterior left nasal packing and numerous sutures on skin wounds were placed. Following the treatment the patient's condition was stable and there was no local bleeding. Seven days after the surgery, sutures were removed, the nasal ala and facial wounds healed properly, the nose was patent, and the cosmetic effect was very good. The patient gave a history of homogenic aortic valve implantation 20 years ago followed by reoperation of an artificial aortic valve implantation 11 years ago. He has been treated for $1 \mathrm{I}^{\circ} \mathrm{cir}-$ culatory insufficiency for 20 years and undergoing longterm treatment with anticoagulant drugs. Ultrasonic abdominal examination had displayed steatosed liver and a $12 \mathrm{~mm} \times 16 \mathrm{~mm}$ lesion of lower echoginicity at the anterior part of the right hepatic lobe resembling an angioma. He had received multiple laryngological treatments due to recurrent bleeding from the body of this tongue. He had undergone repeated surgical ligation of the bleeding lingual blood vessel accompanied by blood transfusion, acenocoumarol dose modification and international normalized ratio (INR) control. We render the presented case extremely challenging as spontaneous haemorrhages in patients

Address for correspondence: Katarzyna Błochowiak DDS, PhD, Department of Oral Surgery, Poznan University of Medical Sciences, 70 Bukowska St, 60-812 Poznan, Poland, phone: +48 6185470 59, e-mail: kasia@naszdentysta.com.pl Received: 5.03.2013, accepted: 25.04.2013. 


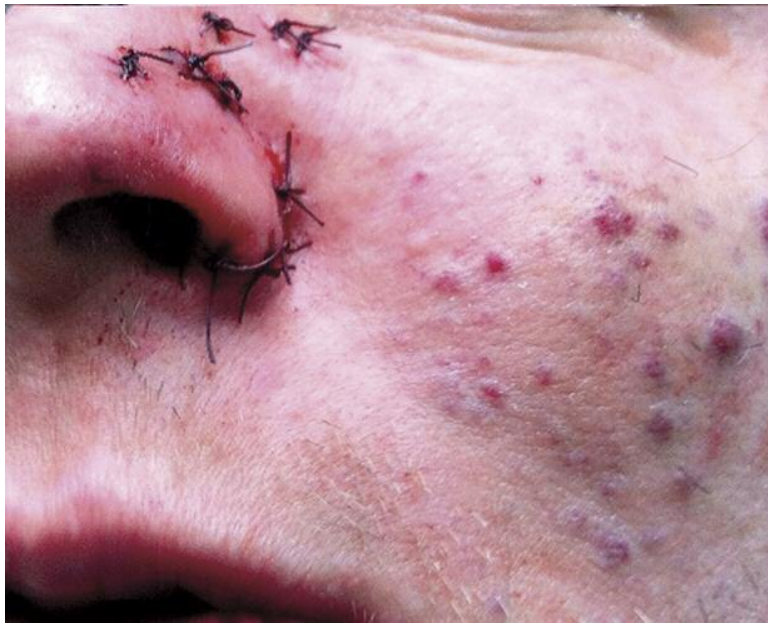

Fig. 1. Vascular lesions on the cheek

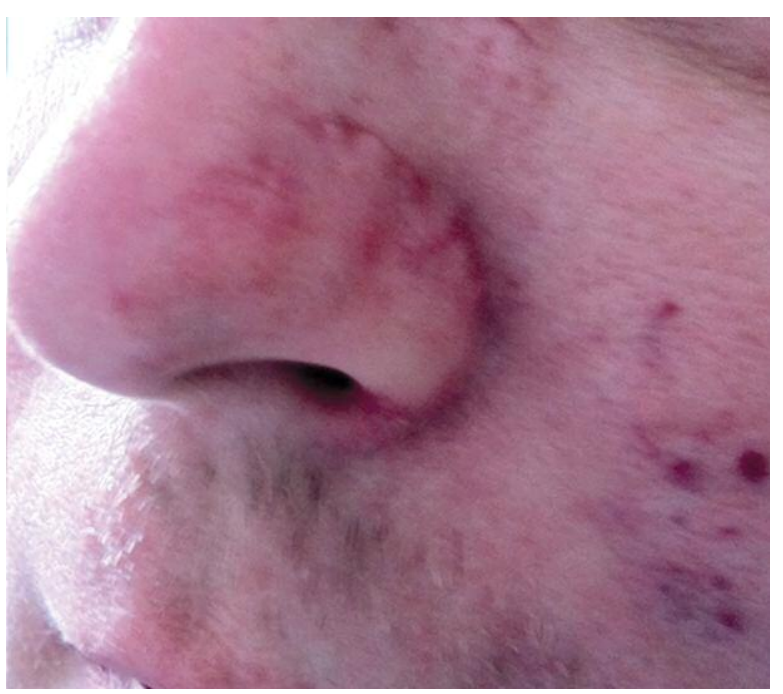

Fig. 2. The nose after plastic reconstruction

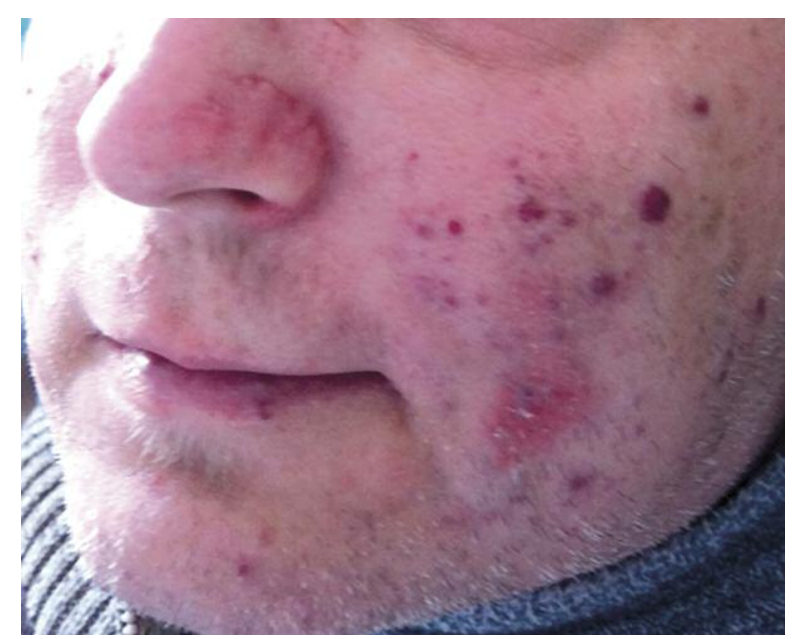

Fig. 3. The patient after nasal reconstruction affected with hereditary hemorrhagic telangiectasia (HHT) pose a difficult therapeutic problem. Together with an injury to the face, complicated by the need of long-term administration of anticoagulants, a HHT patient faces a likely life-threatening haemorrhage (Figures 1-3).

\section{Discussion}

Hereditary haemorrhagic telangiectasia, also called Rendu-Osler-Weber syndrome, is an entity involving numerous arterio-venous malformations. In the classification of vascular abnormalities, which includes slow- and fast-flow malformations, it is considered a slow-flow one. The absence of capillaries between the arterial and venous circulations accounts for a direct contact between these blood vessels and is responsible for spontaneous and recurrent bleedings. The diagnosis of HHT is made basing on the presence of three out of four symptoms: spontaneous epistaxis, skin telangiectasias, arterio-venous malformations in internal organs, and familial character of the disease [1-4]. The clinical picture changes with age. The first symptoms appear in adolescence and are mainly recurrent episodes of epistaxis, often occurring at night. The most predisposed bleeding site is the anterior part of the nose, the middle nasal concha, and the floor of the nasal cavity. The site and morphology of nasal telangiectasias change with age, the applied treatment and nasal septum condition [5-8]. Telangiectasias also affect the skin and mucosa, leading to massive bleedings, following even minor injuries. They are most common in the skin of the face, nose, fingers, auricles, lips vermilion and mucosa, and oral and pharyngeal mucosa. Telangiectasias involved in $\mathrm{HHT}$ diagnosis are not specific. They usually take the form of little red patches, which fade when pressed. In 30\% of patients they appear before the $20^{\text {th }}$ year of life, and in one third before the $40^{\text {th }}$ year of life. They were observed in a 6 -year-old child at the earliest. It has been estimated that $25 \%$ of sufferers present with extra-nasal haemorrhages, which usually are self limiting; still, in $12 \%$ they are prolonged and require treatment. Bleedings most often arise from the base and body of the tongue, like in the presented case, and from the fingers and supraclavicular fossa. The earlier the skin and mucosal lesions appear, the greater the risk of bleedings [9]. A dermatologist is most often the first doctor to diagnose the disease properly, to extend diagnostic procedures onto vital organs, to implement a wide range of treatments and thus prevent later unfavourable and often life-threatening complications. In the case of vascular skin lesions, a meticulous history taking is needed to confirm their familial occurrence accompanied by spontaneous bleedings, especially from the oral cavity, nose, and alimentary tract. Major bleedings from the alimentary tract or the nose are contraindications to the administration of anti-inflammatory and anticoagulant drugs. The history should also reveal possible concurrent pulmonary, cardiac, hepatic and neurological dis- 
eases as well anaemia and polycythaemia. An antibiotic cover is required before every invasive and dental surgery due to the risk of pulmonary arterio-venous malformations. In patients diagnosed with $\mathrm{HHT}$, annual iron level tests should be ordered to prevent anaemia from developing. In HHT patients extended diagnostic procedures include magnetic resonance imaging of the head, ultrasonic examination or computed tomography of the liver, contrast echocardiogram and taking systolic pressure of the pulmonary artery to detect pulmonary hypertension. Differential diagnosis is required between telangiectasias in the Rendu-Osler-Weber syndrome and ataxia-telangiectasia syndrome, congenital benign telangiectasia and vascular lesions found in chronic hepatic disorders. Sequential photocoagulation using argon laser and sclerotherapy provide good effects in arresting bleedings from the oral mucosa $[10,11]$. Unsatisfactory aesthetic effect caused by telangiectasias in the facial skin may be effectively eliminated with a dye laser [12]. At an older age, changes affect internal organs. Twenty five per cent of patients aged 50 years and older develop bleedings from the alimentary tract. Bleedings in the brain, lungs, pancreas and liver are rarer [13]. The presented patient had telangiectasias in the facial skin and oral mucosa as well as recurrent bleedings from the lingual vessels. Vascular lesions of the internal organs were mainly found in the liver. The patient could not state if similar vascular changes were present in other family members. However, he reported spontaneous nasal bleedings in the past. The prevalence of HHT in the young population ranges from $1: 50000$ to 1 : 100000 . Most fatal cases result from diffuse pulmonary, cerebral and digestive tract bleedings. The HHT patients are also diagnosed with haematuria due to slight bleedings and arterio-venous fistulas in the urinary tract. The more severe symptoms of anaemia, the greater likelihood of urinary tract bleeding [14]. Hereditary haemorrhagic telangiectasia is caused by a mutation in the group of genes coding the cascade of TGF- $\beta$ /BMP transmitters, i.e. the ENG, ACVRL 1 (ALK1), SMAD4 genes together with two other genes not yet identified. The HHT is inherited as an autosomal dominant feature. ENG and ACVRL 1 genes mutations are the foundation to identify two HHT types - HHT1 and HHT2, respectively. They differ in character and in sites affected with pathological changes. Oral and nasal mucosa telangiectasias are more frequent in HHT1 than in HHT2. On the other hand, HHT2 patients present with skin lesions more often and at a younger age. In both types the number of changes increases with age. Skin changes, especially those affecting the face, more often occur in HHT1 female patients than HHT1 male ones [15]. The HHT promotes the formation of malignant neoplasms. There are reports of HHT concurrent with malignant neoplasms of the skin, breast, liver, urinary bladder and large intestine $[2,16]$. The treatment of epistaxis involves the application of lubricants, antifibrinolytic drugs, laser ablation, nasal septum dermoplastic surgery and systemic or topical hormones on the nasal mucosa [5-8]. Bleedings from the alimentary tract are treated with endoscopic procedures or segmental surgical resections. In less advanced bleedings hormonal and antifibrinolytic drugs are used [13].

A common problem of HHT sufferers, also present in the described patient, is sideropenic anaemia. Iron preparations are given to prevent its occurrence. Recurrent bleedings often require blood transfusions. Pulmonary or cerebral HHT often necessitates surgical intervention. On the other hand, hepatic vascular changes are often asymptomatic, like in the presented patient, but may eventually lead to irreversible liver damage, its cirrhosis and worsening of the existing circulatory insufficiency.

In conclusion, a patient with typical facial skin telangiectasias should be suspected of having HHT, which was a life-threatening condition in the described case.

\section{References}

1. Lee HE, Sagong C, Yeo KY, et al. A case of hereditary haemorrhagic telangiectasia. Ann Dermatol 2009; 21: 206-8.

2. Gajda S, Szczepanik AM, Misiak A, Szczepanik AB. Excision of the rectum due to carcinoma in patient with hereditary hemorrhagic telangiectasia. Pol Merkuriusz Lek 2011; 31: 45-7.

3. Sabbà C, Gallitelli M, Pasculli G, et al. HHT: a rare disease with a broad spectrum of clinical aspects. Curr Pharm Des 2006; 12: 1217-20.

4. Lenato GM, Guanti G. Hereditary haemorrhagic telangiectasia (HHT): genetic and molecular aspects. Curr Pharm Des 2006; 12: 1173-93.

5. Folz BJ, Wollstein AC, Lippert BM, Werner JA. Morphology and distribution of nasal telangiectasia in HHT-patients with epistaxis. Am J Rhinol 2005; 19: 65-70.

6. Skorek A, Stankiewicz Cz. The problems in diagnosis and management of Rendu-Osler-Weber disease. Otolaryngologia 2010; 9: 155-9.

7. Bruzgielewicz A, Brożek-Mądra E, Osuch-Wójcikiewicz E, et al. Difficulties in the treatment of epistaxis in Rendu-OslerWeber disease. Otolaryngologia 2006; 5: 97-102.

8. Kostrzewska-Poczekaj M, Wróbel M, Rydzanicz M, et al. Analysis of molecular background of hereditary haemorrhagic telangiectasia in Rendu-Osler-Weber disease - preliminary results. Otolaryngologia 2008; 62: 700-4.

9. Folz BJ, Lippert BM, Wollstein AC, et al. Mucocutaneous telangiectases of the head and neck in individuals with hereditary hemorrhagic telangiectasia - analysis of distribution and symptoms. Eur J Dermatol 2004; 14: 407-11.

10. Finkbeiner RL. Progressive laser treatment of hereditary hemorrhagic telangiectasia of the gingival: case report. Int J Periodontics Restorative Dent 2004; 24: 288-94.

11. Hopp RN, de Siqueira DC, Sena-Filho M, Jorge J. Oral vascular malformation in a patient with hereditary hemorrhagic telangiectasia: a case report. Spec Care Dentist 2012; 32: 11-4.

12. Dave RU, Mahaffey PJ, Monk BE. Cutaneous lesions in hereditary haemorrhagic telangiectasia: successful treatment with the tunable dye laser. J Cutan Laser Ther 2000; 2: 191-3.

13. Chamberlain SM, Patel J, Carter Balart J, et al. Evaluation of patients with hereditary hemorrhagic telangiectasia with video capsule endoscopy: a single center prospective study. Endoscopy 2007; 39: 516-20. 
14. Di Gennaro L, Ramunni A, Suppressa P, et al. Asymptomatic microhematuria: an indication of hereditary hemorrhagic telangiectasia? J Urol 2005; 173: 106-9.

15. Letteboer TG, Mager HJ, Snijder RJ, et al. Genotype-phenotype relationship for localization and age distribution of telangiectases in hereditary hemorrhagic telangiectasia. Am J Med Genet A 2008; 1: 2733-39.

16. Kluger N, Riviere S, Coupier I. Association of hereditary haemorhagic telangiectasia (Rendu-Osler-Weber syndrome) and malignant melanoma in two patients. Melanoma Res 2007; 17: 201-3. 